\title{
Digestive Surgery
}

Abou-Shady, M. 140

Agresti, M. 30

Aguilar-Nascimento, J.E. 252

Ahmad, P. 60

Aleksic, M. 342

Alemdaroglu, K. 333

Alexandrescu, V. 266

Andersson, R. 386

Andrei, V.E. 256

Andrén-Sandberg, A. 92

Angeli, E. 410

Armbruster, C. 669

Asanuma, Y. 15

Bach Lausten, S. 287

Baer, H.U. 140, 273, 364, 703 , 709

Balzano, G. 410

Bassi, C. 241

Baxter, P.S. 35

Bayat, M. 665

Beckerhinn, P. 669

Bergh, C.K. 317

Berguer, R. 135

Bicakci, K. 713

Bilbao, J.E. 369

Bittner, R. 167

Blauensteier, W. 669

Blum, H.E. 191

Boghdadi, I. 287

Bota, D. 663

Botsios, D. 693

Boutin, Z. 247

Bratucu, E. 663

Bravo, F. 69

Brier, C. 135

Brisinda, G. 30

Brönnimann, S. 364

Brücher, B.L.D.M. 42

Büchler, M.W. 55, 91, 92, 148, $158,193,364,385$

Bühler, L. 656

Busch, R. 42

Butturini, G. 241

Caldiron, E. 241

Carkman, S. 25

Caulfield, A. 656

Célicout, B. 697

Cenitagoya, G.F. 317

Chan, S.Y.Y. 707

Chenard, X. 247
Cheng, J.-S. 328

Chigot, J.-P. 247

Christensen, K.Ø. 665

Civello, I.M. 30

Cohen, M. 270

Conte, D. 75

Coşar, E. 713

Craxi, G. 64

Cretin, N. 656

Cristaldi, M. 75

Curti, G. 193

Dadoukis, J. 693

Debus, E.S. 260

Demetriades, Ch. 693

Demircan, O. 713

Di Carlo, V. 410

Di Cataldo, A. 64

Eleftheriadis, E. 357

Elli, M. 75

El-Sefi, T. 287

El-Sibai, O. 347

Emparan, C. 369

Endo, M. 12

Erguney, S. 25, 333

Eriksson, S. 683

Ertem, M. 333

Falconi, M. 241

Fan, J. 674

Farmakis, H. 357

Farthmann, E.H. 385

Feodorovici, M.A. 140, 158

Fingerhut, A. 697

Fink, U. 42

Foulis, A.A.K. 72

Fournier, B. 656

French Associations for Surgical Jacobi, C.A. 110 Research 697

Friis-Andersen, H. 665

Fuentes, O. 69

Fujiwara, Y. 404

Fukuda, A. 299

Galandiuk, S. 60,655

Gani, O.S. 372

García, A. 69

Gen, T. 12

Giray, S. 25

Glaser, Ch. 703

Glättli, A. 703, 709

Gutt, C.N. 135

Kidd, M. 209
González, C. 69

Goseki, N. 12

Granström, L. 683

Graziani, R. 241

Greif, F. 52, 270

Gschwantler, M. 669

Gysi, B. 709

Hammarström, L.-E. 323

Hansen, E.S. 665

Hanyu, F. 299

Harada, H. 21

Harada, N. 299

Hata, K. 352

Hatori, T. 299

Haupt, W. 102

Hay, J.-M. 697

Heini, P.F. 185

Hennes, N. 342

Herodotou, A. 357

Himpens, J. 181

Hohenberger, W. 102, 105

Holzinger, F. 273, 709

Нøу, K. 665

Iannucci, A. 241

Ikeuchi, H. 679

Imaizumi, T. 299

Imhof, M. 308

Imrie, C.W. 72

Inoue, A. 227

Ito, K. 12

Itoh, H. 687

Iturburu, I. 369

Iwasa, M. 227

Iwasa, Y. 227

Jankovic, Z.B. 374

Jensen, S.L. 287

Jespersen, S.M. 665

Kaganoi, J. 352

Karahasanoglu, T. 25, 333

Kawarada, Y. 218

Kawasaki, S. 21

Ker, C.-G. 328

Kinoshita, T. 687

Kitamura, H. 21

Klinger-Roitman, J. 317
Kobayashi, A. 21

Köckerling, F. 105

Kotzampassi, K. 357

Koufogiannis, D. 357

Koyama, K. 15

Kraft, K. 167

Krähenbühl, L. 91, 140, 148, 158,185

Kral, J.G. 279

Kriwanek, S. 669

Kumagai, K. 236, 337

Kume, M. 227

Kusano, T. 15

Küssner, C. 260

Kusunoki, M. 404, 679

Kuzinkovas, V. 703

La Greca, G. 64

Lakéhal, M. 224

Landen, S. 297

Latulippe, J.-F. 117

Launois, B. 224

Lee, K.-T. 328

Leibl, B. 167

Lelcuk, S. 52, 270

Leone, B.E. 410

Le Prisé, E. 224

Lesma, A. 75

Levard, H. 697

Licata, A. 64

Li Destri, G. 64

Lindblad, B.E. 665

Liu, Y.-E. 328

López-Cantarero, M. 69

Lottenbach, M. 185

Lu, J.-Z. 674

Ma, Z.-C. 674

Maddern, G.J. 35

Madhala, O. 270

Maeda, H. 227

Majerus, B. 266

Malinverni, R. 364

Man, W.K. 252

Manafis, K. 693

Margolis, M. 256

Maria, G. 30

Masuo, K. 337

Mathie, R.T. 252

Matsuda, S. 218

Matsumoto, K. 687

Maurer, C.A. 193

Fax + 41613061234 
Maurer, Ch. 703

Mehler, C. 102

Mendez, J. 369

Menegaux, F. 247

Mentha, G. 656

Methaste, A. 12

Meunier, B. 224

Mezzabotta, M. 75

Minelli, S. 30

Mittelkötter, U. 260

Miyagawa, S. 21

Mizumoto, R. 218

Modlin, I.M. 209

Morel, Ph. 656

Mortensen, F.V. 665

Mouton, W.G. 703

Msika, S. 697

Müller, J.M. 110

Naef, M. 709

Nakagawa, K. 404

Nakayama, N. 352

Nishioka, A. 227

Nishitai, R. 352

Noike, T. 21

Nomura, K. 21

Oberholzer, J. 656

Ogawa, Y. 227

Ogoshi, S. 227

Ogura, Y. 218

Ohmann, C. 308

Ohmori, Y. 227

Ohsumi, K. 352

Ordemann, J. 110

Ortiz, J. 369

Osborne, A.W. 707

Osman, M. 287

Öttinger, A. 145

Ozcelik, F. 25

Ozkan, S. 713

Paksoy, M. 25

Paladugu, R. 417
Palermo, A. 30

Paolucci, V. 124, 135

Pederzoli, P. 241

Pelissier, E. 697

Pesko, P.M. 374

Poon, F.W. 72

Prinz, R.A. 716

Procacci, C. 241

Puleo, St. 64

Purkiss, S.F. 707

Qiu, S.-J. 674

Ranstam, J. 323

Raoul, J.-L. 224

Rashed, M.-Y. 287

Rau, A. 417

Reber, P.U. 92

Redaelli, C.A. 55

Reichen, J. 1

Reina, A.J. 69

Reith, H.B. 260

Renzulli, P. 140

Reymond, M.A. 105

Riemer, V. 135

Riese, J. 102

Rinzivillo, C. 64

Roder, J.D. 42

Röher, H.-D. 308

Rotestein, Z. 52

Rovati, M. 75

Rubin, M. 52

Rucinski, J.C. 256

Sabat, R. 110

Sahin, M. 72

Sakurai, H. 218

Salvia, R. 241

Sánchez, B. 69

Sarr, M.G. 398, 716

Sasaki, N. 15

Sato, T. 15

Schaeff, B. 124

Schäfer, M. 140, 148, 158
Schein, M. 256, 417

Schilling, M.K. 55, 273, 376

Schippers, E. 145

Schlageter, M.-O. 372

Schmedt, C.-G. 167

Schmied, B. 92

Schneider, C. 105

Schröders, C. 308

Schumpelick, V. 145

Schwarz, J. 167

Schwarzenbach, O. 185

Sclafani, S.J.A. 279

Seki, H. 21

Senturk, H. 25

Sganga, G. 30

Shafik, A. 347

Shah, I.A. 372

Sheen, P.-C. 328

Shimada, R. 21

Shimizu, K. 337

Shimonov, M. 52

Shindo, Y. 15

Shoji, Y. 679

Shutsha, E. 266

Siewert, J.R. 42

Sönmez, H. 713

Sontheimer, J. 315

Stain, S. 273

Stein, D.E. 279

Stein, H.J. 42

Styrud, J. 683

Sun, Z. 386

Suzuki, H. 687

Tanaka, T. 337, 404

Tang, L.H. 209

Tang, Z.-Y. 674

Taschieri, A.M. 75

Thiede, A. 260

Thomopoulos, J. 124

Thompson, J. 153

Timmermans, M. 266

Tittel, A. 145

Tokunaga, Y. 352
Tonouchi, H. 687

Torné, P. 69

Tsalis, K. 693

Tsiotos, G.G. 398

Ulmeanu, D. 663

Ulrich, B. 342

Uras, C. 333

Utsunomiya, J. 404

Vago, L. 75

Van Ruyssevelt, Ch. 266

Veronesi, P. 410

Volk, T. 110

Wang, X. 386

Watson, A. 172

Weber, K. 102

Wechsler, B. 247

Wenger, F. 110

Wexner, S.D. 117

Wibin, E. 266

Williamson, R.C.N. 252

Wise, L. 256, 417

Wong, S.-R. 328

Wong, S.X. 372

Wu, Z.-Q. 674

Yamagata, K. 337

Yamamoto, A. 227

Yamamura, T. 404, 679

Yanagi, H. 679

Yoshida, S. 227

Yu, Y.-Q. 674

Zamboni, G. 241

Zerbi, A. 410

Zeren, S. 713

Z'graggen, K. 273

Zhou, J. 674

Zhou, X.-D. 674

Zimmermann, H. 1

Zisiadis, A. 693

Zowe, M. 102 